\title{
Detailed simulation of distance protection for its testing and setting
}

\author{
Nikolay Yu. Ruban, Mikhail V. Andreev, Ruslan A. Ufa, \\ Aleksey A. Suvorov, Alexandr S. Gusev*
}

\begin{abstract}
A significant part of severe accidents (blackouts) in electric power systems (EPS) is associated with incorrect operation of relay protection and automation (RPA). One of the main reasons for the incorrect actions of the RPA devices is its rough settings, which often does not correspond to the real operating conditions for specific device. An analysis of currently used methods and tools for RPA setting up, shown that they are largely relied on the guidelines of previous decennaries. Respectively modern techniques have the same drawbacks associated with accounting the processes in specific RPA and primary transducers and its errors by approximate coefficients. It is possible to solve the indicated problem with a highly detailed analysis of the operation of key elements of RPA schemes in the specific operating conditions. The obtained results allow to estimate the processes in protected objects, processing errors in instrumental current (ICT) and voltage (IVT) transformers, as well as in RPA itself. Such possibility could be achieved by the detailed RPA mathematical modeling. The combination of an adequate EPS simulator and RPA models allows configuring parameters of the RPA settings ensuring its correct operation in real EPS. The article presents result of this research for distance protection.
\end{abstract}

K e y w or d s: hybrid real-time power system simulator, distance protection, power system, mathematical simulation

\section{Nomenclature}

$\mathrm{ACT} / \mathrm{AVT}$ - auxiliary current/voltage transformer;

$\mathrm{CC}$ - comparison circuit;

DP - distance protection;

EPS - electric power system;

FACTS - flexible alternating current transmission system;

FAP - former of the anticoincidence pulses;

FCV - former of the compared values;

HRTSim - Hybrid Real-Time Power System Simulator;

HVDC - high-voltage direct current technology;

ICT/IVT - instrumental current/voltage transformer;

$\mathrm{MU}$ - memory unit;

NRP - numerical RPA device;

PT - primary transducer;

RES - renewable energy source;

$\mathrm{RPA}$ - relay protection and automation;

TE - tripping element;

$\mathrm{TF}$ - transfer function.

\section{Introduction}

The adequate operation of relay protection and automation (RPA) in modern electric power systems (EPS) is still not properly solved, despite its obvious importance. It is particularly confirmed by statistics of severe accidents in EPS [1]. According to the statistics - a quarter of all blackouts are caused by incorrect actions of the
RPA devices. One of the main reasons for the incorrect relay protection operation is its 'rough' setting up (low sensitivity), which often does not correspond to the real operating conditions of specific relay protection device [2]. It is associated with approximate fixed coefficients application in used methods. In addition, they are completely inappropriate for modern EPSs those contain a significant part of renewable energy sources (RES), distributed generation, FACTS and HVDC devices. This conclusion is confirmed, for example, by the publications $[3,4]$. In these articles, the authors develop algorithms for determining the RES volume and location in such a way that it does not affect the RPA settings and, consequently, their operation.

The main purpose of this article is to solve mentioned above problem and to develop new methods and means of the RPA setting up, ensuring their adequate operation in all modes of modern and prospective EPS.

We have determined two interrelated factors for inadequate RPA settings calculation, as a result of the analysis of existing methods, fully corresponding to distance protection (DP):

- Usage of incomplete and in some cases unreliable information about modes and processes in EPS.

- Taking into account processes in specific RPA and primary transducers (PT) as well as its impact on signal processing by approximate fixed coefficients.

The first factor is the same for any RPA type and connected with the problem of adequate EPS simulation [5-

* School of Energy \& Power Engineering, Tomsk Polytechnic University (TPU), Lenina Avenue, 30, 634050, Tomsk, Russia, rubanny@tpu.ru 
8]. But at present, there are software and hardware EPS simulators eliminating this factor, for example, Hybrid Real-Time Power System Simulator (HRTSim) [9]. Because of it the tasks of RPA in-depth research, testing and setting become solvable and urgent. In the opinion of the authors and according to the available results [10,11], the tasks can be solved through a detailed analysis of relay protection devices operation under specific operating conditions due to possibility of reliable assessment of errors of PT and RPA' elements. Based on these results, it becomes possible to determine the adequate RPA settings and to identify the causes of RPA incorrect actions.

The analysis of the current RPA simulation methods is carried out. As a result, it was identified that in projects devoted to the assessment of the EPS stability the RPA models are not reproduced or simplified to reproduce only the main functions: comparison with a threshold, time delaying and sending command for switching devices. Such situation is connected with necessity of modeling a largescale EPS. In the works concentrated mainly on the RPA simulation, the authors have revealed the following approaches:

- Reproduction of processes in electromechanical, induction and electronic relays using a system of algebraic and differential equations $[12,13]$. Detailed mathematical models of these relays individually are known and available in open sources. The authors did not found mathematical models of these relays combination in a whole RPA system and research results of its operation in EPS model.

- Simulation of operation algorithms of the RPA: this approach is applied to study of modern numerical (microprocessor) RPA devices (NRP). In some cases, NRP' mathematical models are limited to simulate only the digital part [14-16], which implements the algorithm of its operation. In others, measuring part is partly taken into account [17]: instrumental current (voltage) transformers and analog frequency filters are modelled.

The authors $[10,11]$ formulated the approach for development of RPAs detailed mathematical models. It is described in details in Section 2. The main difference between this approach and existing [12-19] is the creation of a single mathematical description (in the form of a transfer function (TF) and corresponding system of differential equations) simultaneously for the whole set of elements contained in particular RPA device: instrumental transformers, auxiliary converters, frequency filters, relays, etc. Such approach allows taking into account all interconnections in a natural way, which increases the reliability and adequacy of simulation results by eliminating potential errors in a case of scheme equivalenting. Authors call such approach 'all-regimes simulation' of RPA.

The above simplifications are dictated by the general RPA simulation trend. In particular, in the papers [1416], the authors do not take into account the auxiliary transformers. In their opinion, it does not effect on the adequacy of the simulation results. As for analog frequency filters (in most cases - low-pass filters), there is uncertainty in their choice, since detailed information for the NRP is not available in the firm guidelines. In this case, according to the literature sources, there are several types of analog filters that can potentially be used in NRP: 1) Butterworth third-order low-pass filter [18]; 2) Butterworth fourth-order low-pass filter; 3 ) Chebyshev low-pass filter, Bessel low-pass filter and elliptic low-pass filter [19]. However, the authors did not found publications containing specific data confirming the validity of the conclusions about the insignificance of these elements' influence on the NRP operation. The results of the study carried out and shortly presented in $[10,11]$ lead to other conclusions. In these publications shown that the effect of auxiliary transformers on the operation of a specific relay protection is significant. Additionally, this is confirmed by studies results for the DP, the fragments of which are presented below.

Speaking about DP the first, second or third zones of the DP (if operational, automatic or tele-acceleration is available) can operate without time delay or with a minimum time delay - during the transient in the EPS. Imposition of unfavorable conditions can lead to the development of local faults to blackouts, because of false DP tripping [20]. It could be nature disasters, breakdowns or incorrect operation of other RPA devices, incorrect actions of personnel and other factors. Taking into account this possibility, it is important to simulate such operating conditions of real EPS. In addition, it should be noted that the RPA, including DP, operate in a wide spectrum of processes in equipment and EPS as a whole in emergency modes, mostly asymmetric, often with a frequency deviation, currents and voltages form distortion. Moreover, the harmonics spectrum can be quite wide, and their presence, according to [21], has a significant effect on the operation of the protection tripping element.

The impact of PT and specific elements of DP on controlled signals processing is accounted by generalized coefficients with fixed values in accordance with currently used settings guides [22]. The coefficients could be specified with dynamic simulation of processes in PT and all key elements of particular DP in normal, emergency and post-emergency operating modes. The methodology of detailed DP models development is presented below.

Using the model allows to determine exactly how the process is going on in a specific part of the device and what needs to be done to make its settings more adequate to the real conditions of operation. The software and hardware complex can reproduce the process in the power system with sufficient accuracy, but it is not always possible to find out the cause of incorrect operation, and the software and hardware complex only confirms the device's tripping or failure. To analyze causes of relay incorrect operation it is necessary to test its various elements, which requires the use of additional devices and is not always possible. A model is flexible and convenient tool for such tasks. 


\section{The methodology of development of detailed distance protection mathematical models}

The problem solution of DP all-regimes simulation with taking into account specific implementations and PT includes the following steps:

\subsection{Synthesis of detailed mathematical models}

(1) Analysis of schemes and technical data of DP and PT

As a result of this analysis, two groups of functional elements can be formed, which must be taken into account in simulation

- microprocessor and microelectronic based elements: analog-to-digital, digital-to-analog converters, microprocessors and etc, high precision of which guarantee its negligibly small hardware error;

- elements used for continuous processing of analog controlled signals, which are mostly based on electromagnetic principle: PTs, various auxiliary transformers used in current and voltage circuits, filters, circuits for forming and comparing special (zero sequence, negative sequence and etc) analog signals.

All listed elements of RP and PTs are modeled as a single scheme. With this approach, all interactions are taken into account in a natural way.

(2) Simulation of functional elements

Functional elements of the first group are described by logical functions and algebraic equations, the characteristics of its hardware implementation are taken to be ideal.

Functional elements of the second group are described by differential equations. The trajectory of obtaining these equations has the following logic: firstly, transfer functions (TF) synthesis, which allows analyzing the DP elements operation in the time and frequency domains; secondly, formation of differential equations on the basis of the corresponding TFs.

TFs determination is performed by the flow graphs method [23] on the basis of equivalent circuits.

(3) Investigation of frequency characteristics and processing error

Investigation of the TFs is used for specifying elements of the equivalent circuits, which have significant and negligible effect on the signals processing errors. As a result of this analysis it is possible to simplify the TF and reduce the differential equation order.

Investigation of the TFs includes the following steps

- analyzing frequency response;

- specifying effect of parameters of simulated functional DP elements on processing errors, depending on various factors according to the sensitivity theory [24] .

(4) Tabulated reproduction of nonlinear magnetization characteristics of electromagnetic elements

According to the magnetization curve $B=f(H)$, which is reproduced as an array of values for particular type of steel of current transformer core, the dependence of which is formed by $L_{\mathrm{sat}}=f\left(i_{\mathrm{sat}}^{\prime}\right)$, the saturation current $i_{\text {sat }}^{\prime}$ is determined by $i_{\text {sat }}^{\prime}=l_{\text {core }} H / w_{2}$ where $l_{\text {core }}$ is the length of magnetic path, $w_{2}$ is the number of turns of secondary winding and $H$ is the magnetic field intensity.

(5) Development of the DP detailed mathematical model

The TF of the PT and DP key elements, as differential equations, logical functions, and tabulated dependencies, describing nonlinear parameters, form detailed RPA mathematical model.

\subsection{Implementation of detailed mathematical models of $D P$}

The developed DP mathematical models, taking into account its specific performances and PTs, are could be applied as a program and solved by one of the numerical methods $[7,8]$. Another way is hardware and software realization.

There are two options for entering the information about EPS processes and modes to the DPs application tools

- Integration into EPS simulators

The application tools can be integrated into existing EPS software and hardware simulators (for example, HRTSim). In this case, input signals are instantaneous currents and voltages continuously obtained from EPS model during simulation process.

\section{- Autonomous tool}

In this case, as input signals can be used the currents and voltages obtained with disturbance recorders or EPS simulators.

\section{Synthesis of the distance protection mathematical model}

According to the given methodology, the detailed DP mathematical model is synthesized taking into account its particular structure and PT. The synthesis of the model of one of the most widespread in Russia DP on the microelectronic basis SHDE-2801 was performed.

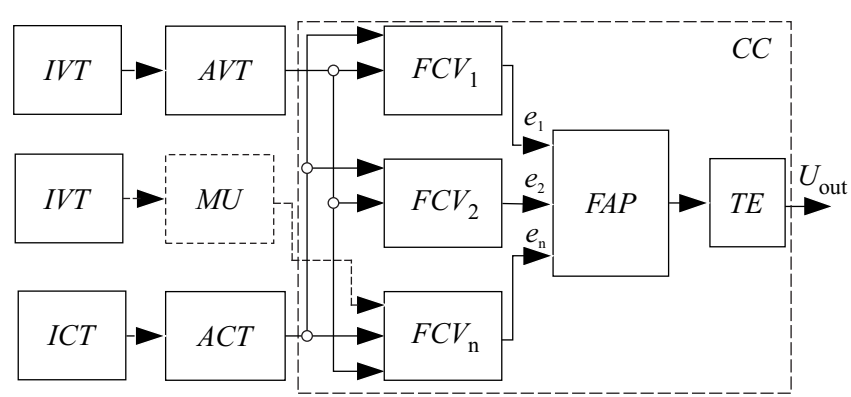

Fig. 1. The block diagram of the DP 


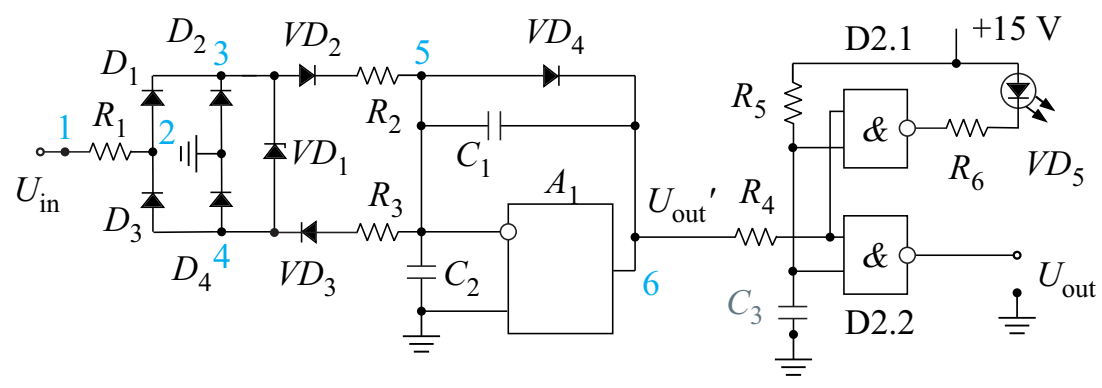

Fig. 2. The schematic diagram of the TE2
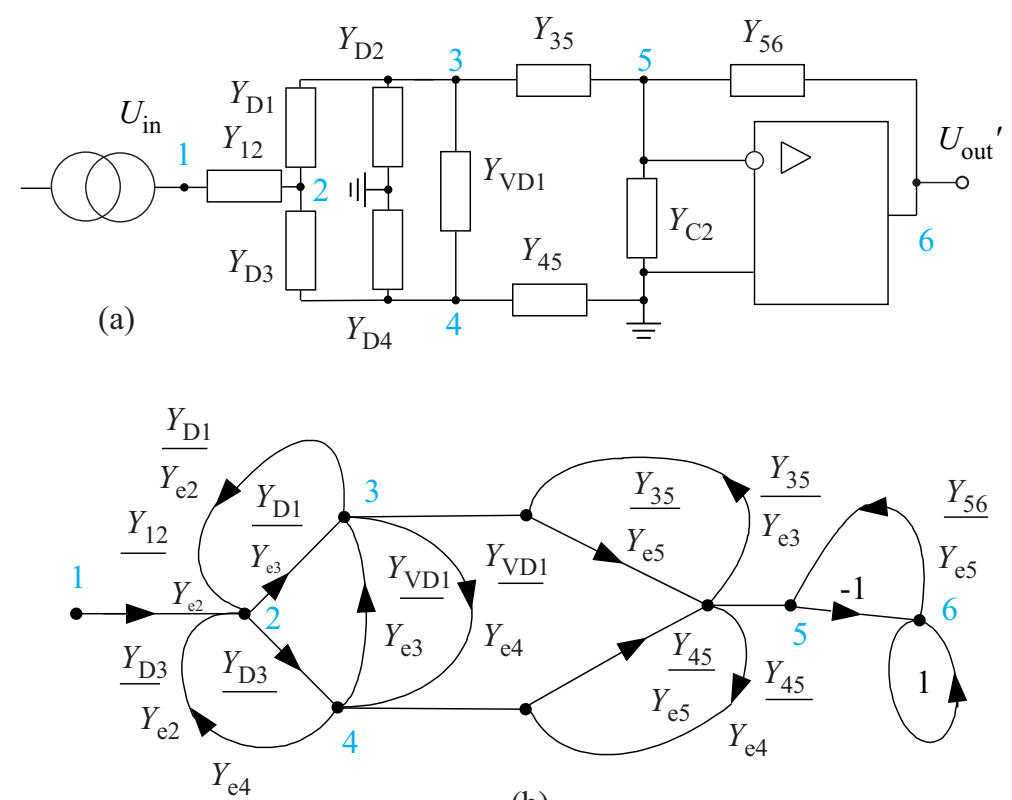

(b)

Fig. 3. The TE2: (a) - equivalent circuit, (b) - flow graph

\subsection{The block diagram of the DP}

The DP performed on a microelectronic element base, usually applies the principle of comparing the phases of two, three or four formed values. The main functional elements used in microelectronic DP are combined in block diagram (Fig. 1) which includes: instrumental current transformer (ICT) and instrumental voltage transformer (IVT); auxiliary current transformer (ACT) and auxiliary voltage transformer (AVT); formers of the compared values $(\mathrm{FCV})$, the former of the anticoincidence pulses (FAP) and the tripping element (TE), which form the comparison circuit (CC). In addition, there may be used a memory unit (MU) in the DP scheme of such structure and with circle mho characteristic passing through the origin of the impedance plane. It is used for the correct DP' functioning in a case of close faults.

This DP structure provides the possibility of forming $n$ values $e_{1}, e_{2}, \ldots, e_{n}$ compared by phase, which in turn makes it possible to realize mho characteristic of various shapes.

In SHDE-2801, a three-zones DP is realized, the connection is made to the difference between the phase currents and the corresponding line voltages.

\subsection{The mathematical model of the DP}

The complete mathematical model of the SHDE-2801 is a large system of differential equations, and therefore, for clarification, the principle of DP models synthesis is further illustrated by the example of one of the main elements of this protection - the tripping element.

Two types of TE (TE1 and TE2) are used in the SHDE-2801. The TE1 compares the discrepancy time, which is equal to the duration of the negative input pulse, with the given signal. The TE1 is used to form polygonal mho characteristic of the second and third zones of the DP. The TE2 compares the discrepancy time, which is equal to the duration of the negative input pulse, with the coincidence time, which is equal to the duration of the positive input pulse. The TE2 is used to form mho characteristic in the form of a circle, an ellipse, or composite mho characteristic formed by the intersection of several circles. In the SHDE-2801 TE2 is used as tripping element of the first zone.

Schematic diagram of the TE2 (Fig. 2) includes a diode assembly, comparator A1, two logical elements "AND" (D2. 1 and D2. 2) . All these elements are described by logical functions. The diode assembly consists of diodes D1, D2, D3, D4, Zener diode VD1, which are taken into 


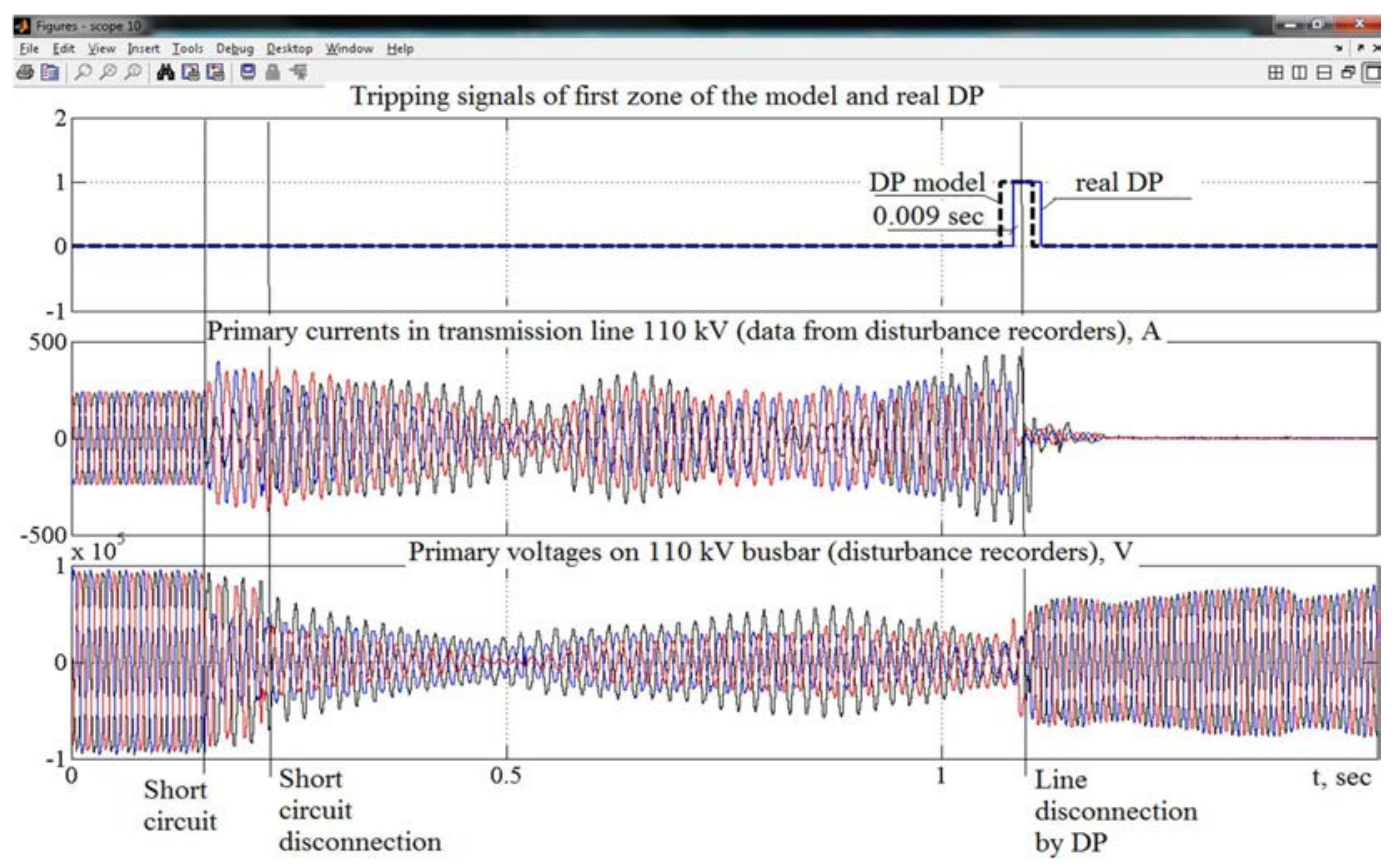

Fig. 4. The results of comparing the operation of the model and the real DP

account in the transfer function by direct or reverse resistance in depends on the polarity of the input pulse. The flow graph of the TE2 is shown in Fig. 3.

The following symbols are used in Figs. 2 and 3 $R_{1}, \ldots, R_{4}$ - resistors; $R_{D 1}, \ldots, R_{D 4}, R_{V D 1}, \ldots, R_{V D 4}$ - resistivities of diodes $D_{1}, \ldots, D_{4}$ and $V D_{1}, \ldots, V D_{4}$; $Y_{D 1}, \ldots Y_{D 4}$ - conductivities of diodes $D_{1}, \ldots, D 4 ; Y_{V D 1}$ - conductivity of Zener diode $V D_{1} ; Y_{12}$ - conductivity of the resistor $R_{1} ; Y_{35}$ - equivalent conductivity of seriesconnected diode $V D_{2}$ and resistor $R_{2}, Y_{45}$ - equivalent conductivity of series-connected diode $V D_{3}$ and resistor $R_{3}, Y_{56}$ - equivalent conductivity of diode $V D_{4}$ and capacitor $C_{1}$ connected in parallel; $Y_{C 2}$ - capacitor $C_{2}$ conductivity; $U_{\mathrm{IN}}$ - input voltage of the $T E_{2} ; U_{\mathrm{OUT}}^{\prime}$ - output voltage of the integrating element of the $T E_{2} ; U_{\text {OUT }}-$ output voltage of the logic element of the $T E_{2}$ (1 or 0$)$; $Y_{e 2}, \ldots, Y_{e 5}$ - equivalent conductivities of nodes $2-5$ of the $T E_{2}$ equivalent circuit.

Equations for the determination of equivalent conductivities are

$$
\begin{gathered}
Y_{35}=\left(Y_{V D 2}+Y_{R 2}\right) / Y_{V D 2} \cdot Y_{R 2} \\
Y_{45}=\left(Y_{V D 3}+Y_{R 3}\right) / Y_{V D 3} \cdot Y_{R 3} \\
Y_{56}=Y_{V D 4}+Y_{1}, \quad Y_{e 2}=Y_{12}+Y_{D 1}+Y_{D 3} \\
Y_{e 3}=Y_{D 1}+Y_{D 2}+Y_{V D 1}+Y_{35} \\
Y_{e 4}=Y_{D 3}+Y_{D 4}+Y_{V D 1}+Y_{45} \\
Y_{e 5}=Y_{35}+Y_{45}+Y_{56}+Y_{C 2}
\end{gathered}
$$

The results of the TF determination of the TE2 are not given in the article due to its large size. In general form, the TF of the TE2 looks as

$$
W_{T E 2}(p)=\frac{B_{1}}{A_{2} p+B_{2}}
$$

where $B_{1}, A_{2}, B_{2}$ are variable coefficients determined by RLC-parameters of equivalent circuit.

The differential equation describing the process in $\mathrm{TE} 2$, in general form, is

$$
A_{2} \frac{\mathrm{d} u_{\mathrm{OUT}}^{\prime}(t)}{\mathrm{d} t}+B_{2} u_{\mathrm{OUT}}^{\prime}(t)-B_{1} u_{\mathrm{IN}}(t)=0 .
$$

The time delay used in the second and third DP zones is realized by a logic function

if TE output signal is ' 1 ', then 'start timer', else 'stop and reset timer'.

\section{Research results}

This section presents the results of an experimental study of the developed in the MATLAB Simulink detailed DP mathematical model of the SHDE-2801 (hereinafter DP model) widely used for the overhead lines protection (110-220 kV). Experimental studies were carried out in order to verify the adequacy of the developed DP model.

\subsection{DP model integrated into MATLAB Simulink}

In accordance with the methodology given in Section 2, experimental studies of the DP model in the MAT$\mathrm{LAB}$ are performed using arrays of instantaneous values of currents and voltages of the protected object of the EPS. In the study the data obtained from disturbance recorders installed in EPS were used in the COMTRADE format. In this case the processes in PT are already taken into account. Thus the PTs are excluded from the aggregate DP model and replaced by the transformation ratio. 


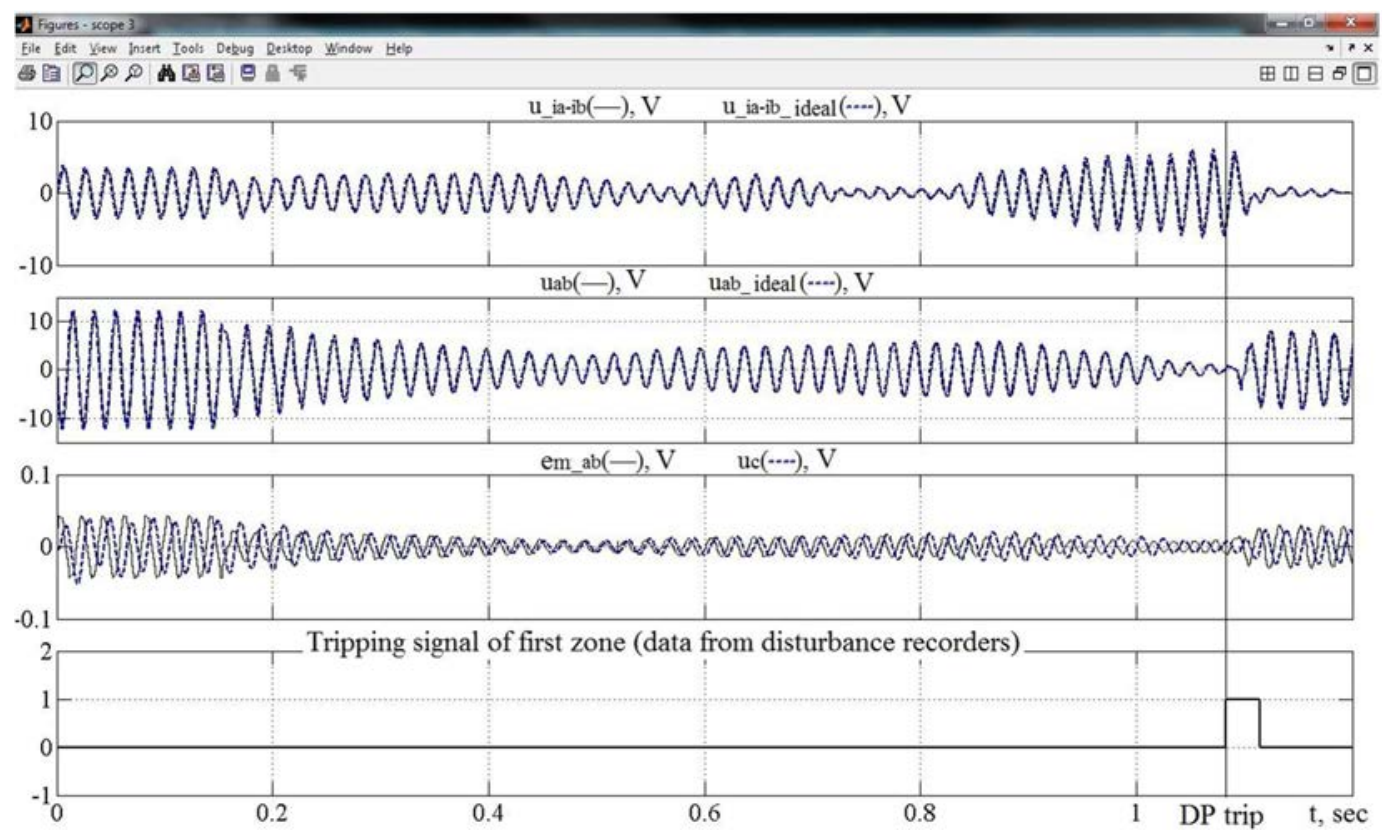

Fig. 5. Oscillograms of the act, AVT and MU of the impedance relay set AB functioning in the swing mode

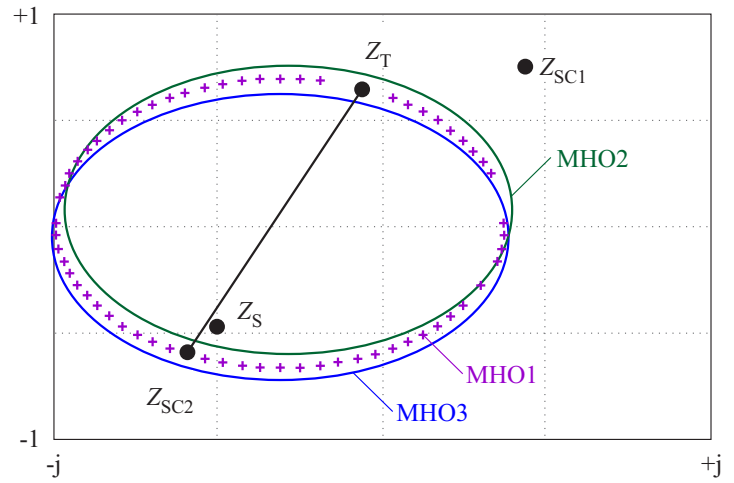

Fig. 6. Mho characteristics of impedance relay set of the first zone of the shde- 2801

The results of its operation are compared with the results of the real DP operation for analyzing the case: false tripping of the SHDE-2801 first zone in the swing mode. The results of the experimental studies are shown in Figs. 4-7.

According to the oscillograms shown in Fig. 4, in the swing mode, the set of the DP first zone protecting phases A and B tripped falsely. The difference in the relay operating time of the model and the real DP is about 0.009 seconds, therefore, the DP model is adequate and its settings reliably reflect the settings of the real DP. Thus, the developed DP model can be used as a tool for analyzing the reasons for the false tripping of a real DP: the functioning of a falsely tripped set of impedance relay is investigated.

The analysis of the following elements functioning is carried out for the impedance relay set AB: ACT, AVT, MU, FCV, FAP and the TE2. The research results of the DP elements operation and the processes in them are given in comparison with operation of the first zone of the real DP for their analysis clarity.

Figure 5 illustrates the almost complete coincidence of the secondary ACT $\left(u_{-} i a-i b\right)$ and the AVT $(u a b)$ voltages with the idealized signals $u \_i a-i b \_i d e a l$ and $u a b \_i d e a l$. Idealized signals are formed by replacing the ACT and AVT models by its transformation ratio. Consequently, these functional elements in the studied mode do not introduce significant errors and do not affect the correct operation of the simulated DP.

In normal mode or remote short-circuit mode, the voltage value $e_{m}$ at the output of the MU is much smaller than the formed values being compared, therefore it does not affect the operation of the DP. In the mode of close three-phase short-circuit, the em decays during several periods, which ensures the DP action by memory. For the correct operation of the first zone of the DP in the close two-phase short-circuit mode, the $e_{m}$ of the impedance relay set, switched on the phase-to-phase voltage of the damaged phases, must coincide in phase with the voltage of the third undamaged phase. In the swing mode at the time of the DP operation, according to Fig. 4, the $e_{m}$ formed in the $\mathrm{MU}$ are commensurable in magnitude with the voltages in the secondary circuits of AVT, hence the influence of these quantities must be taken into account when analyzing the operation of the DP. The MU voltage $e_{m}$ of the analyzed resistance relay set $\mathrm{AB}$ differs in phase from $u_{c}$, with which it must coincide in phase with a close short-circuit, by approximately 180 degrees. This leads to a shift of the mho characteristic not to the third, as is required for close short-circuit, but to the first quadrant of the complex plane.

Figure 6 shows the Mho characteristic of the developed mathematical model of the impedance relay set $\mathrm{AB}$ of the first zone of DP SHDE-2801 and the impedances of the 


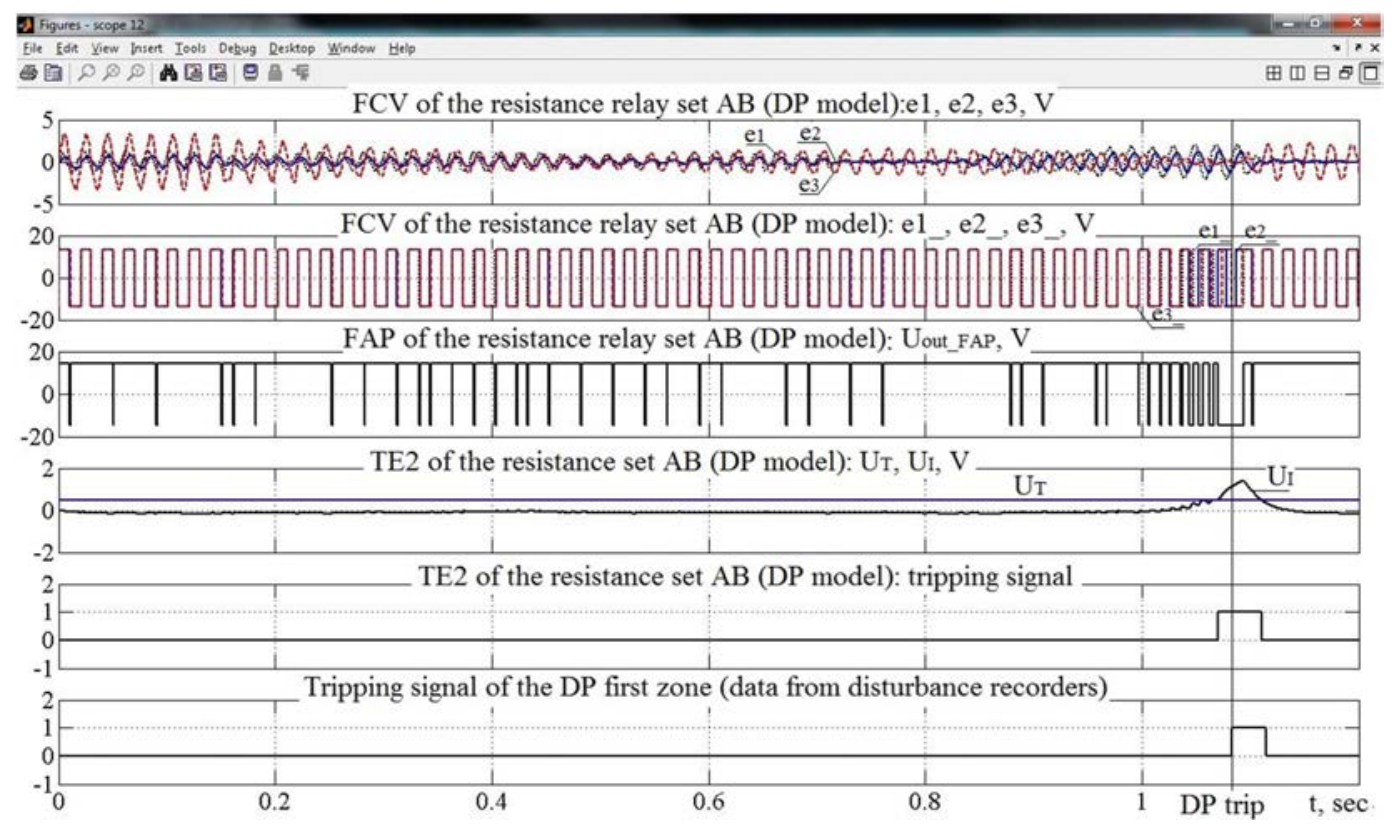

Fig. 7. Oscillograms of the FCV, FAP and TE2 of the resistance relay set AB functioning in the swing mode

protected $110 \mathrm{kV}$ overhead line obtained in the Mathcad program.

The following symbols are used in Fig. 6: $Z_{\mathrm{T}}-$ impedance of the DP first zone threshold; $Z_{\mathrm{SC} 1}$ - impedance of the protected overhead line in a case of three-phase short-circuit on the substation buses, which is opposite from the DP installation site; $Z_{\mathrm{SC} 2}$ - impedance of the protected overhead line in a case of two-phase shortcircuit at the beginning of the line; $Z_{\mathrm{S}}$ - impedance of the protected overhead line at the moment of the DP first zone operation in the swing mode; MHO1 - characteristic corresponds to the three-phase short-circuit mode at the end of the line; MHO2 - characteristic corresponds to the considered swing mode; MHO3 - characteristic corresponds to the two-phase short-circuit mode at the beginning of the line.

According to the results of the studies shown in Figs. 5 and 6 , the operation of the MU affects the shift of the mho characteristic, therefore, if the MU is set up incorrectly, a misoperation can occur in a case of close faults or a false tripping in a case of remote faults. In this swing mode, the MU operates correctly. The false tripping has occurred for another reason - as a result of the DP first zone operation: $\mathrm{ZS}$ is defined as the ratio of the phaseto-phase voltage $u_{a b}$ to the difference of currents $i_{a}-i_{b}$, and is located in the zone MHO2 of the impedance relay set $\mathrm{AB}$ at the moment of the DP operation (Fig. 6).

This conclusion is confirmed by oscillograms illustrating the operation of FCV, FAP and TE2 in the considered swing mode, which are shown in Fig. 7.

The following symbols are used in Fig. 7: $e_{1}, e_{2}, e_{3}$ - oscilograms of sinusoidal output signals of amplifierslimiters of the FCV; $e_{1_{-}}, e_{2_{-}}, e_{3_{-}}-$output pulse signals of amplifiers-limiters of the FCV; $u_{\text {out_FAP }}$ - output signal of the FAP; $u_{\mathrm{T}}$ - voltage threshold of the TE2; $u_{\mathrm{I}}$ output voltage of the integrating element of the TE2.

According to the principle of the DP action, it can operate falsely in the swing mode without an appropriate swing blocking device. The results of researches confirm that the impedance relay set AB operates correctly. False tripping occurred as a result of the fact that the measured values of controlled parameters were corresponded to the settings of this set. From the foregoing it follows that the reason for the false tripping of the real SHDE-2801 is either the malfunction of the swing blocking device or its inefficiency in the studied mode.

Thus, based on the results of the pilot studies, the following activities are recommended: (i) an extra check of the used swing blocking device together with the SHDE2801; (ii) in the case of the swing blocking device serviceability, it is expedient to check its effectiveness in the swing mode. Checking efficiency of the swing blocking device is possible by means of its mathematical modeling according to the methodology proposed in this paper and studying its operation in the considered swing mode and in the spectrum of other possible modes, modelled, for example, with the help of HRTSim. If it is not possible to carry out the above studies and to determine the specific reasons for the ineffectiveness of the swing blocking device, it is recommended, for example, to replace the used DP and swing blocking devices with a modern type, for example, on a microprocessor-based within the framework of modernization of the substation.

\section{2 model integrated into EPS Simulator}

The programming codes realizing the DP model were integrated into EPS simulator - HRTSim.

This experiment is as follows. Integrated in the HRTSim DP model used as the main protection from phase-to- 

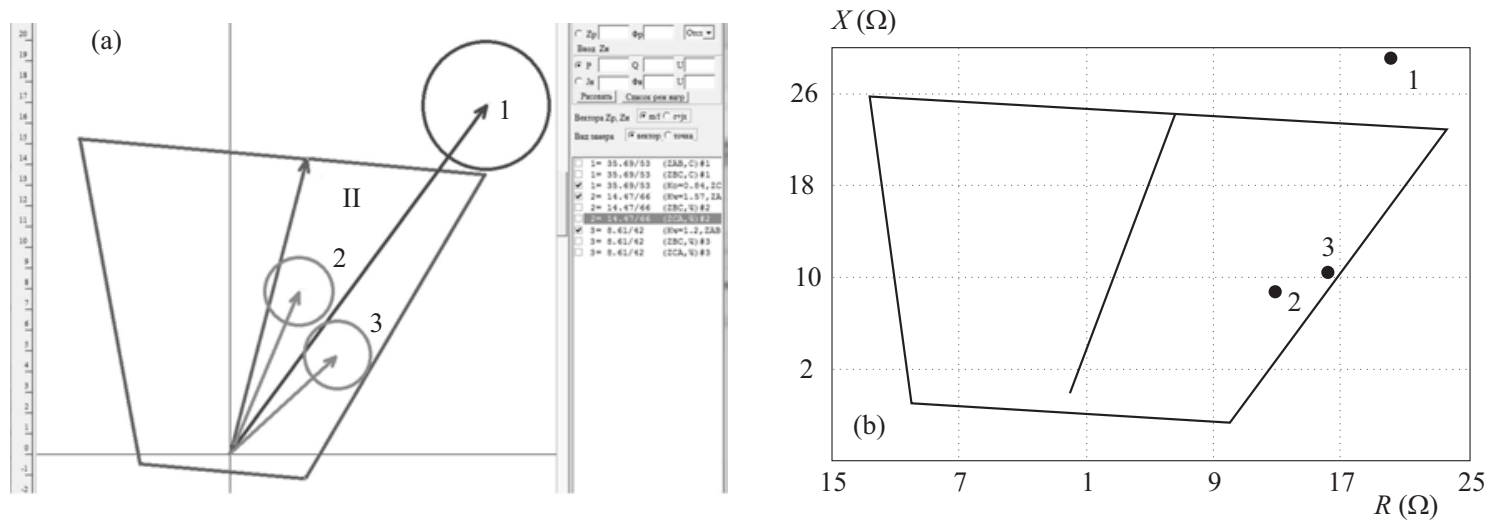

Fig. 8. Comparison of the operating characteristics of the second zone of the DP installed at substation B in a case of three-phase short circuit close to substation A: (a) - DP model in ARM SRZA, (b) - DP model in HRTSim

phase short circuits of the $110 \mathrm{kV}$ transmission line (simulated in the HRTSim) connecting the substations A and B. The experiment was carried out for the case of a threephase short circuit close to Substation A. Simulation results allow obtaining information for analysis and correction of the DP setting and confirm the adequate functioning of the developed model.

Figure 8 shows the operating characteristics of the second zone of the DP installed at the substation B under the mentioned short-circuit case. The characteristic of the DP shown in Fig. 8(a) is carried out using software for mathematical simulation of EPS - ARM SRZA (Russia) based on symmetrical component method for short-circuit currents calculation. The characteristic shown in Fig. 8(b) is carried out using integrated in the HRTSim DP model.

In the characteristics shown in Fig. 8, point 1 corresponds to the impedance of the DP first zone of the next line. That is needed for DPs coordination. Point 2 corresponds to the impedance of the protected line before the line disconnection by the first zone of the DP installed at substation A. Point 3 corresponds to the impedance after disconnection of the line by the 1st zone of the DP installed at substation A. Since point 3 was in the frameworks of the characteristic of the second zone of the DP installed at substation $\mathrm{B}$, disconnection of the line from this side was also successful. However, it can be seen in Fig. 8 (b) that the point 3 is practically on the boundary of the operating characteristic in the mode after the line disconnection from the side of substation A. Therefore, in order to ensure the reliability of the operation of the second zone of the DP installed at substation B in this mode, it is advisable to shift the right boundary of the operating characteristic to the right (two positions of the right boundary of the second zone are provided in the simulated DP type SHDE-2801).

\section{CONCLUSION}

The validity and the practical relevance of the developed DP mathematical models are confirmed by the following solved tasks: 1) provided the detailed analysis of the DP operation in specific conditions; 2) determined the parameters for an adequate setting of the DP on the basis of the results of this analysis.

Using EPS simulators (for example, HRTSim), it is possible to simulate a wide spectrum of emergency regimes of EPS, which have not yet been recorded by disturbance recorders, but potentially could take place in a particular EPS. Such modes (for example, short-circuit regimes, characterized by flowing very high currents, overvoltage regimes, etc) cannot be reproduced in a real EPS due to known negative effects.

The data obtained with such simulation can be used for:

- researching the impact of PT and functional elements of DP and determining its signal processing errors,

- researching hardware and algorithmic features of the particular implementation of DP,

- setting DPs in operating conditions, close to real.

As a result of the research by the HRTSim the functioning of the DP model, taking into account errors of instrument transformers and elements of the DP, the possibility of specifying the DP settings in comparison with the traditional methodology has been determined.

The mentioned approach could be applied for modeling microprocessor-based relay protection. For microprocessor distance protection work is still underway and the results will be published in the future.

\section{Acknowledgements}

This work was supported by the Ministry of Education and Science of the Russian Federation under the governmental grant "Science" No. 13.5852.2017/8.9 (Development of the concept for comprehensive validation of calculating modes and processes in electric power system and tools of its realization)

\section{REFERENCES}

[1] J. Sykes, V. Madani, J. Burger, M. Adamiak and W. Premerlani, "Reliabilty of Protection Systems (What are the Real Con- 
cerns)", Proc. Protective Relay Engineer Conf., College Station, TX, USA, 2010, pp. 1-16.

[2] K. K. Li, L. L. Lai and A. K. David, "Stand Alone Intelligent Digital Distance Relay", IEEE Transactions on Power Systems, vol. 15, no. 1, Feb 2000, pp. 137-142.

[3] H. H. Zeineldin, H. M. Sharaf, D. K. Ibrahim, Essam, El-Din, Abou and El-Zahab, "Optimal Protection Coordination for Meshed Distribution Systems with DG Using Dual Setting Directional Over-Current Relays", IEEE Transactions on Smart Grid, vol. 6, no. 1, Jan 2015, pp. 115-123.

[4] H. Zhan, C. Wang, Y. Wang, X. Yang, X. Zhang, C. Wu and Y. Chen, "Relay Protection Coordination Integrated Optimal Placement, Sizing of Distributed Generation Sources Distribution Networks", IEEE Transactions on Smart Grid, vol. 7, no. 1, Jan 2016, pp. 55-65.

[5] G. Hall and J. M. Watt, "Stiff problems", Modern Numerical Methods for Ordinary Differential Equations, Oxford, UK: Clarendon Press, 1976, pp. 123-208.

[6] J. C. Butcher, "Differential, Difference Equations", Numerical Methods for Ordinary Differential Equations: Early Days the Birth of Numerical Analysis, 2nd ed., Hoboken, New Jersey, USA: John Wiley \& Sons, 2008, pp. 1-49.

[7] N. Watson and J. Arrillage, "Numerical Integrator Substitution", Power Systems Electromagnetic Transients Simulation, London, UK: The Institution of Engineering, Technology, 2003, pp. 67-98.

[8] E. Hairer and G. Wanner, "Examples of Stiff Equations", Solving Ordinary Differential Equations: Stiff, Differential Algebraic Problems, vol. 14, Germany, Berlin: Springer, 1996, pp. 2-14.

[9] A. Prokhorov, Yu. Borovikov and A. Gusev, "Real Time Hybrid Simulation of Electrical Power Systems: Concept, Tools, Field Experience, Smart Grid Challenges," Int. J. Smart Grid Clean Energy, vol. 1, no. 1, Sep 2012, pp. 67-68.

[10] M. V. Andreev, A. O. Sulaymanov and A. S. Gusev, "Simulation of Differential Protections of Transformers Power Systems", Proc. DPSP, Edinburgh, UK, 2016, pp. 1-6.

[11] M. V. Andreev, A. S. Gusev, A. O. Sulaymanov and Y. S. Borovikov "Setting of Relay Protection of Electric Power Systems using its Mathematical Models, 2017 IEEE Innovative Smart Grid Technologies Conference Europe (ISGT-Europe): conference proceedings, Turin, September 26-29, 2017. New York: IEEE, 2017, pp. 1-6.

[12] Z. Peng, M. S. Li, C. Y. Wu, T. C. Cheng and T. S. Ning, "A Dynamic State Space Model of a mho Distance Relay", IEEE Trans. on Power Apparatus, Systems, vol. PAS-104, no. 12, Dec 1985, pp. 3558-3564.

[13] A. Domijan and M. V. Emami, "State Space Relay Modeling, Simulation using the ElectroMagnetic Transients Program, its Transient Analysis of Control Systems Capability", IEEE Transactions on Energy Conversion, vol. 5, no. 4, Dec 1990, pp. 697-702.

[14] C.-S. Chen, C.-W. Liu and J.-A. Jiang, "Application of Combined Adaptive Fourier Filtering Technique, Fault Detector to Fast Distance Protection", IEEE Transactions on Power Delivery, vol. 21, no. 2, Apr 2006, pp. 619-626.

[15] K. Seethalekshmi, S. N. Singh and S. C. Srivastava, "A Classification Approach using Support Vector Machines to Prevent Distance Relay Maloperation under Power Swing, Voltage Instability", IEEE Transactions on Power Delivery, vol. 27, no. 3, July 2012, pp. 1124-1133.

[16] V. H. Makwana and B. R. Bhalja, "A New Digital Distance Relaying Scheme for Compensation of High-Resistance Faults on Transmission Line", IEEE Transactions on Power Delivery, vol. 27, no. 4, Oct 2012, pp. 2133-2140.

[17] M. R. Dadash Zadeh, T. S. Sidhu and A. Klimek, "FPAA-based mho Distance Relay Considering CVT Transient Supervision", IET Generation, Transmission \& Distribution, vol. 3 no. 7, July 2009, pp. 616-627.
[18] M. Kezunovic and Q. Chen, "A Novel Approach for Interactive Protection System Simulation", Proc. Conference and Exposition on Transmission and Distribution, Los Angeles, USA, 1998, pp. 458-464.

[19] S. G. Perez, M. S. Sachdev and T. S. Sidhu, "Modeling Relays for Use in Power System Protection Studies", Proc. Canadian Conference on Electrical and Computer Engineering, Saskatoon, Canada, 2005, pp. 566-569.

[20] G. Andersson, P. Donalek, R. Farmer, N. Hatziargyriou, I. Kamwa, P. Kundur, N. Martins, J. Paserba, P. Pourbeik, J. Sanchez-Gasca, R. Schulz, A. Stankovic, C. Taylor, V. Vittal, "Causes of the 2003 Major Grid Blackouts in North America and Europe, and Recommended Means to Improve System Dynamic Performance", IEEE Transactions on Power Systems, vol. 20, no. 4, Oct 2005, pp. 1922-1928.

[21] Z. Zexin, S. Xiaofan, Z. Chunxia, W. Shirong, J. Yiguo and D. Dingxiang, "Dynamic Simulation Inspection Test for Protection Equipment in China", Proc. PowerCon, Kunming, China, 2002, pp. 280-284.

22] IEEE Guide for Protective Relay Applications to Transmission Lines, IEEE Standard SA-C37.113-2015, 2015.

[23] J. R. Abrahams and G. P. Coverley, "Operations with a Flow Graph", Signal Flow Analysis, 1st ed., Oxford, UK: Pergamon press, 1965, pp. 31-46.

[24] P. Eykhoff, "Some Fundamental Aspects of Process Parameter Estimation", IEEE Trans. Automat. Contr., vol. AC-8, no. 4, , Oct 1963, pp. 347-357.

Received 31 March 2018

Nikolay Yu Ruban received his Engineer and PhD degrees in 2010 and 2014 at Tomsk Polytechnic University. Currently he is an associate professor of Division for Power and Electrical Engineering of School of Energy \& Power Engineering of Tomsk Polytechnic University. His research interests include simulation of smart grids, control systems, relay protection and automation.

Mikhail V. Andreev received his Engineer and PhD degrees in 2010 and 2013 at Tomsk Polytechnic University. Currently he is head of Power Grid Simulation Lab of School of Energy \& Power Engineering of Tomsk Polytechnic University. His research interests include simulation of smart grids, control systems, relay protection and automation.

Ruslan A. Ufa received his MSc in 2012 at Tomsk Polytechnic University. Currently he is lecturer of Division for Power and Electrical Engineering of School of Energy \& Power Engineering of Tomsk Polytechnic University. His research interests include simulation of power systems based on HVDC system and smart grids.

Aleksey A. Suvorov received his Engineer in 2014 at Tomsk Polytechnic University. Currently he is a PhD student and teaching assistant of Division for Power and Electrical Engineering of School of Energy \& Power Engineering of Tomsk Polytechnic University. His research interests include simulation electric power systems and validation of electric power system simulation tools.

Alexandr S. Gusev received the DSc (Tech) degree in electrical engineering from Tomsk Polytechnic University, Tomsk, Russia, in 2008. He is a Professor of Division for Power and Electrical Engineering of School of Energy \& Power Engineering of Tomsk Polytechnic University. His main fields of interest are hybrid simulation technology of power system and smart grids. 